\title{
The Urokinase Receptor Is Required for Human Monocyte Chemotaxis In Vitro
}

\author{
Margaret R. Gyetko, * Robert F. Todd III, ${ }^{\star}$ Camille C. Wilkinson, * and Robert G. Sitrin * \\ * Division of Pulmonary and Critical Care Medicine, and ${ }^{\ddagger}$ Division of Hematology/Oncology, Department of Internal Medicine, Ann \\ Arbor Veterans Affairs Medical Center and University of Michigan Medical Center, Ann Arbor, Michigan 48109-0360
}

\begin{abstract}
Mononuclear phagocytes (M $\phi$ ) produce urokinase-type plasminogen activator (UPA) and also express a specific cell-surface receptor for urokinase, uPAR. The concomitant expression of these proteins provides a mechanism by which $\mathrm{M} \phi$ can degrade extracellular matrix proteins during directed cell migration. In this study, we sought to determine if uPAR plays a role in $M \phi$ chemotaxis that is distinct from its role in matrix proteolysis. Exposing adherent monocytes to a chemotactic gradient causes plasma membrane uPAR to localize strongly to the leading edge of cell migration. Adherence alone or exposure to FMLP had no effect on uPAR expression. Using Boyden chamber chemotaxis assays, we demonstrate that treating mononuclear cells with an anti-uPAR mAb (either as an intact mAb or $\left.F\left[a b^{\prime}\right]_{2}\right)$ ablates chemotaxis induced by FMLP and monocyte chemotactic peptide-1 $(P<0.001)$. Inactivating the catalytic activity of uPAR-bound uPA had no effect on chemotaxis. Similarly, blocking uPAR expression with an antisense oligonucleotide to uPAR completely ablates chemotaxis, but blocking uPA expression with an antisense oligonucleotide to uPA has a minimal effect. We therefore demonstrate that expression and unimpeded function of UPAR plays an obligate role in $\mathbf{M} \phi$ chemotaxis by mechanisms that are largely independent of its ligand, uPA. Combined with its known role in mediating pericellular proteolysis, these observations demonstrate that UPAR is essential for both locomotion and traversing tissue barriers during M $\phi$ migration. (J. Clin. Invest. 1994. 93:1380-1387.) Key words: macrophage - mononuclear phagocyte $\bullet$ plasminogen activators $\bullet$ motility $\bullet$ antisense oligonucleotides
\end{abstract}

\section{Introduction}

For monocytes to be successfully recruited to inflammatory sites, at least two distinct functions are required. First, they must accomplish directional migration in a chemotactic gradient. This complex process requires a sequence of adhesion, cytoskeletal rearrangement, movement, and detachment steps $(1,2)$. Second, cells often traverse tissue planes, a process that may require expression of extracellular proteases to degrade specific extracellular matrix $(E C M)^{1}$ proteins (3-5). One

Address correspondence to Margaret R. Gyetko, M.D., 3916 Taubman Center, Medical Center Drive, Ann Arbor, MI 48103-0360.

Received for publication 24 August 1993 and in revised form 12 November 1993.

1. Abbreviations used in this paper: $\mathrm{Ab}$, antibody; AS, antisense; ECM, extracellular matrix; GPI, glycosyl phosphatidylinositol; HMW, high molecular weight; $\mathbf{M} \phi$, mononuclear phagocyte; NS, nonsense; oligo, oligonucleotide; rMCP-1, human recombinant monocyte chemotactic peptide-1; S, sense; uPA, urokinase-type plasminogen activator; uPAR, UPA receptor.

The Journal of Clinical Investigation, Inc.

Volume 93, April 1994, 1380-1387 mechanism by which leukocytes can promote ECM degradation is by converting plasminogen to plasmin through the action of urokinase-type plasminogen activator (uPA) $(6,7)$. Mononuclear phagocytes ( $\mathbf{M} \phi)$ produce uPA and also express specific high affinity cell-surface receptors for UPA (uPAR) (4, 7-9). This receptor allows uPA to function as a membrane-associated ectoenzyme, thereby limiting proteolysis to the immediate pericellular environment $(4,10)$. Both $\mathbf{M} \phi$ and a variety of malignant cells have been shown to use uPAR-associated uPA to degrade matrix proteins during tissue invasion $(3,11$, 12). Although ECM degradation requires catalytically active UPA, it is not known whether UPA and UPAR are involved in other mechanisms necessary for cell movement.

Several intriguing observations support a role for UPAR in cell locomotion. In malignant cells, uPAR localizes to cellular binding sites to ECM, where it colocalizes with cytoskeletal components such as vinculin (13-15). This suggests that uPAR may participate in a transmembrane structure that links the ECM with the cell surface and cytoskeleton. Increased UPAR and UPA expression has recently been described in migrating endothelial cells (16). One antibody to UPAR inhibits $\mathbf{M} \phi$ responsiveness to migration inhibition factor, providing another potential association between UPAR and cell movement (17). The uPA-uPAR system is known to affect leukocyte behavior by mechanisms unrelated to plasminogen activation. UPA can directly cleave ECM components and can act as a chemotaxin for neutrophils and as a mitogen for lymphocytes (18-20). Also, uPA and plasmin play important roles in modulating cytokine release and activity $(21,22)$. A portion of the uPA molecule, the growth factor domain, may trigger $\mathbf{M} \phi$ differentiation and adhesion by binding to UPAR (23).

In this study, we sought to determine if uPAR plays a role in cellular migration that is distinct from its participation in ECM proteolysis. We show that human monocytes alter the plasma membrane distribution of UPAR on exposure to a chemotactic gradient, as uPAR becomes tightly localized to the leading edge of migration. Adhesion on plastic alone and exposure to FMLP have no effect on total uPAR expression. We also demonstrate that functional UPAR is required for mononuclear cell chemotaxis under ECM-free conditions, as chemotaxis is ablated when cells are pretreated with anti-uPAR monoclonal antibody or antisense-uPAR oligonucleotides. Finally, we show that expression or catalytic activity of UPA is not required for chemotaxis under these conditions. We therefore provide the first conclusive evidence that the expression and unimpeded function of UPAR is an obligate factor in the directional migration of mononuclear phagocytes in response to a chemotaxin.

\section{Methods}

Reagents. High molecular weight urokinase (HMW-uPA) and murine anti-human uPA mAb were obtained from American Diagnostica Inc. (Greenwich, CT). This antibody neutralizes uPA catalytic activity and recognizes free UPA, receptor-bound UPA, and pro-uPA (24). LPS 
from Escherichia coli 0111:B4 was purchased from Sigma Chemical Co. (St. Louis, MO). An FITC-conjugated goat anti-mouse $F\left(a^{\prime}\right)_{2}$ antibody $(\mathrm{Ab})$ was obtained from Cappel Laboratories (Durham, $\mathrm{NC}$ ). The mouse anti-human uPAR mAb (clone 3B10, $\operatorname{IgG}_{2 \mathrm{a}}$ ), also designated anti-Mo3f, was prepared as previously described and recognizes an epitope in the UPAR ligand-binding region (domain 1) (25, 26). A series of IgM anti-uPAR mAbs included anti-Mo3d (clone 100 ), anti-Mo3c (clone 68), and anti-Mo3e (clone 109) (17, 27). These mAbs were shown to bind to uPAR-transfected 3T3 cells (Garni-Wagner, B., and R. F. Todd III, unpublished observations). Control antibodies included an anti-MHC class II mAb (clone 9-49, $\mathrm{IgG}_{2 \mathrm{a}}$ ) (28); an anti-Mo5 $\mathrm{mAb}$ (a monocyte antigen of unknown function) (clone 99, $\operatorname{IgG}_{2 a}$ ) (29), and two anti-CD1 lb mAbs (clone 44, IgG2a; and clone 94, IgM) (30). Anti-uPAR and control mAbs were used as dilutions of ascites, while anti-uPAR $F\left(a b^{\prime}\right)_{2}$ fragments and anti-uPA were used as purified IgG, quantitated by protein content.

Cell purification and culture. Human PBMC were purified from buffy coats provided by the American Red Cross (Detroit, MI). Buffy coats were diluted 1:1 with $5 \mathrm{mM}$ EDTA/normal saline and purified by density gradient centrifugation through Lymphoprep ${ }^{\mathrm{TM}}$ (Nycomed Pharma, Oslo, Norway). After washing with RPMI 1640 (Gibco Laboratories, Grand Island, NY), cells were counted in a hemocytometer, and viability was assessed by trypan blue exclusion. Differential cell counts were determined by examining Giemsa-stained cytocentrifuge samples. The mononuclear cells were resuspended in serum-free medium MAC-SFM (Gibco Laboratories) supplemented with penicillin $(100 \mathrm{U} / \mathrm{ml})$, streptomycin $(100 \mu \mathrm{g} / \mathrm{ml})$, gentamicin $(100 \mu \mathrm{g} / \mathrm{ml})$, and L-glutamine ( $2 \mathrm{mM}$ ).

For some experiments, monocytes were purified by adherence in 35-mm plastic dishes (Corning Inc., Corning, NY) at $2 \times 10^{7}$ cells/ well ( $\sim 8 \times 10^{6}$ monocytes) for $1 \mathrm{~h}$ in humidified air containing $5 \%$ $\mathrm{CO}_{2}$ at $37^{\circ} \mathrm{C}$. Nonadherent cells were removed by washing with RPMI 1640 at $37^{\circ} \mathrm{C}$. Monocytes were then detached by gentle scraping with a rubber spatula, resuspended in MAC-SFM, and cultured for $24 \mathrm{~h}$ either in 60-mm plastic petri dishes (Corning) or 55-mm Teflon dishes (Scientific Specialties Service Inc., Randallstown, MD), as indicated.

Antisense oligonucleotide ( $A S$-oligo) inhibition of gene expression. AS-oligos represented the reverse complement of high specificity sequence regions. The AS-oligos for UPA and UPAR correspond to nucleotides 426-444 and 720-738, respectively $(31,32)$. Controls included oligonucleotides of random sequence and length, sense (S)uPAR, and nonsense (NS)-uPAR (same base composition of AS-uPAR, but in random order). AS-, S-, and NS-oligos were synthesized by the DNA Synthesis Core of the University of Michigan on automated DNA synthesizers, substituting sulfur for oxygen at the phosphate moiety. These phosphorothioate oligonucleotides are nuclease resistant (33). Cells were incubated with $30 \mu \mathrm{M}$ AS-oligos for 24 $\mathrm{h}$ at $37^{\circ} \mathrm{C}$. Efficacy of AS-oligo treatment was confirmed by demonstrating loss of immunofluorescent staining for the corresponding protein. Cell viability exceeding $95 \%$ was confirmed by trypan blue exclusion before using AS-oligo-treated cells in experiments.

Immunolabeling. BMC or purified monocytes were resuspended in staining buffer (PBS with $1 \%$ BSA and $0.1 \%$ sodium azide, pH 7.4). Immunolabeling for UPA or UPAR was performed by incubating the cells with either an anti-uPA or anti-uPAR primary $\mathrm{mAb}$ for $30 \mathrm{~min}$, $4^{\circ} \mathrm{C}$, followed by labeling for $30 \mathrm{~min}, 4^{\circ} \mathrm{C}$, with FITC-conjugated goat anti-mouse $F\left(a b^{\prime}\right)_{2}$ secondary $A b$. Negative control cells were stained with the secondary $\mathrm{Ab}$ alone or with an irrelevant isotype-matched primary $\mathrm{Ab}$. For some experiments, the same labeling procedure was used for fluorescent microscopy of glass-adherent monocytes.

Immunofluorescent flow cytometric analysis. Cells were selected by gating ( $\log$ forward angle versus log right angle light scatter). Immunofluorescent intensity was assessed as a measure of relative antigen expression using a flow cytometer (EPICS; Coulter Corp., Hialeah, FL) with a logarithmic amplifier. The channel number ( $\log$ scale) representing the mean fluorescent intensity (major fluorescent peak) was determined from $\sim 5,000$ cells. The corresponding linear fluorescent intensity channel was then calculated from a logarithmic-linear calibration formula. Specific fluorescence intensity was calculated by subtracting the values of the negative controls using a linear scale, and the result was reconverted to log scale.

Chemotaxis assay. PBMC were suspended in Teflon dishes to prevent adherence. For experiments using mAb-pretreated cells, cells were incubated with $\mathrm{mAbs}$, as indicated, in azide-free buffer for $30 \mathrm{~min}$ at $4^{\circ} \mathrm{C}$. For experiments using oligonucleotide-treated cells, cells were incubated with $30 \mu \mathrm{M}$ oligos for $24 \mathrm{~h}$ at $37^{\circ} \mathrm{C}$. Where indicated, cells were incubated with exogenous HMW-uPA $(4 \mu \mathrm{g} / \mathrm{ml})$ at $4^{\circ} \mathrm{C}$ for $30 \mathrm{~min}$. After pretreatment, cell viability was confirmed by trypan blue exclusion, and the cells were washed, resuspended in media, and loaded into the upper chambers of a multiwell chemotaxis apparatus $\left(1 \times 10^{6}\right.$ cells/well), separated from either $10^{-7}$ M FMLP (Sigma Chemical Co.) or $50 \mathrm{ng} / \mathrm{ml}$ recombinant human monocyte chemotactic peptide1 (rMCP-1; Genzyme Corp., Cambridge, MA) by a single $5-\mu \mathrm{m}$ pore $50-\mathrm{mm}^{2}$ polyvinylpyrrolidone-free filter, and incubated at $37^{\circ} \mathrm{C}$ for 2 $h$. The filters were then removed, and the upper surface was scraped free of cells, fixed in methanol, and stained with toluidine blue. The number of cells that had migrated through the pores was counted for each condition. Data are reported as number of cells counted per high power field (three to five fields for each condition) and expressed as percentage of control cells that were not treated with antibody or oligonucleotide. Controls for the chemotaxis assay included medium without chemotaxin in both the upper and lower chambers (random cell movement) and equal concentration of chemotaxin in both upper and lower chambers (chemokinesis).

Statistics. Comparisons between groups were performed using a single sample two-tailed Student's $t$ test (34). Data are expressed as mean percentage of untreated control cells \pm SEM. $n$ is the number of donors, each providing cells for one experiment.

\section{Results}

Effects of a chemotactic gradient on the cell-surface distribution of $U P A R$. Adherent monocytes were exposed to a chemotactic gradient created by placing a $25-\mu$ l bead of FMLP $\left(10^{-7} \mathrm{M}\right)$ at one spot on the slide. After $30 \mathrm{~min}$, the cells were stained with anti-uPAR mAb followed by an FITC-conjugated secondary $\mathrm{Ab}$ and examined by fluorescent microscopy (Fig. 1). Negative control cells were stained with the secondary Ab alone. Untreated monocytes (not exposed to FMLP) were relatively dull, and the distribution of cell-surface UPAR was homogeneous. However, when the monocytes were exposed to an FMLP gradient, staining for uPAR became granular and strongly localized to the leading edge facing the chemotactic gradient in approximately one-third of the cells. When cells were stained for uPA, a similar pattern of localization towards the chemotactic gradient was observed (not shown). Clustering of surface uPA has been reported previously in 30\% of FMLP-exposed monocytes (10). Thus, monocytes cluster UPAR and endogenously generated uPA toward an area corresponding to the leading edge of cell migration. It is presently unclear whether clustering of uPAR and uPA occurs, at least transiently, in all migrating cells.

To determine if FMLP stimulation itself affects the quantity of UPAR on the plasma membrane, monocytes were exposed to FMLP for 30 min after adherence to plastic. After incubation, the cells were gently released and immunolabeled, and uPAR expression was quantitated by flow cytometry. Exposure to a range of concentrations of FMLP $\left(10^{-7}-10^{-11} \mathrm{M}\right)$ did not alter the overall expression of uPAR on the monocyte cell surface relative to control cells (mean fluorescence of control, 121 17 ; FMLP $10^{-7} \mathrm{M}, 116 \pm 9$; FMLP $10^{-11} \mathrm{M}, 121 \pm 15$; $P=$ not significant $)$. In similar experiments, we evaluated the 


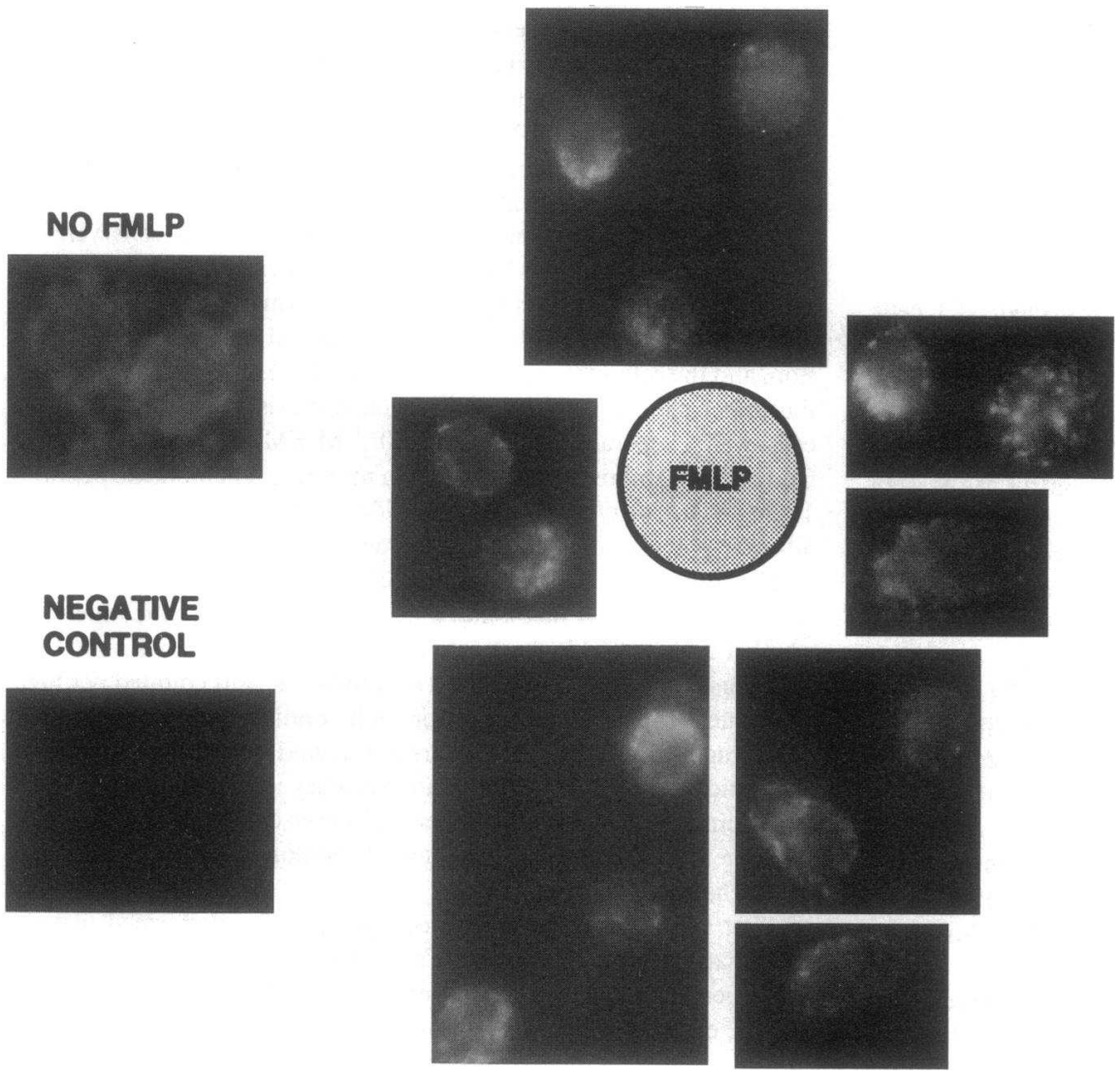

Figure 1. Effects of exposure to an FMLP gradient on monocyte plasma membrane distribution of uPAR. Glass-adhered monocytes were cultured in the presence and absence of an FMLP gradient, stained for surface uPAR by indirect immunofluorescence, and visualized by fluorescent microscopy. Cells cultured in the absence of FMLP are dull, with homogeneous staining (upper left). Cells exposed to an FMLP gradient show granular staining and strong localization of UPAR to the leading edge of the cell oriented toward the FMLP bead (center). effects of adherence on monocyte surface expression of UPAR. Monocytes were cultured either adherent to plastic petri dishes or nonadherent in Teflon dishes. The cells were then removed from culture and immunolabeled, and surface uPAR expression was determined by flow cytometry. UPAR expression was unaffected by adherence to plastic (plastic 91 \pm 8.7 , Teflon 88.8 $\pm 4.9, P=$ not significant $)$.

As we were examining membrane redistribution of UPAR in response to FMLP, we attempted to immunolabel uPAR before adhering the monocytes to slides and exposing them to an FMLP gradient. These anti-uPAR-pretreated cells did not exhibit clustering of UPAR toward the chemotactic gradient (not shown). This suggested to us that the anti-uPAR mAb (3B10) interacts with UPAR in a way that prohibits normal UPAR clustering in response to a chemotactic gradient. We therefore questioned whether this anti-uPAR mAb also affected the ability of these cells to migrate toward a chemotactic gradient.

Effects of anti-uPAR and neutralizing anti-uPA $\mathrm{mAb}$ on mononuclear cell chemotaxis. We sought to determine whether functional uPAR or catalytically active uPA were required for monocyte chemotaxis in a standard Boyden chamber in the absence of serum and ECM, using FMLP $\left(10^{-7} \mathrm{M}\right)$ as the chemotaxin. Antibodies were titered to determine optimal binding to monocytes, as determined by flow cytometry. AntiUPAR and isotype-matched control mAbs were used at a 1:50 dilution, and anti-uPA was used at $5 \mu \mathrm{g} / 10^{7}$ cells per milliliter. Mononuclear cells were incubated in medium alone or were pretreated with either anti-uPAR or anti-uPA mAb before resuspension in medium. The Boyden chambers were incubated for $2 \mathrm{~h}$ at $37^{\circ} \mathrm{C}$. The number of mononuclear cells that tra- versed the filter in response to the chemotactic gradient was expressed as the percentage of control cells that did not receive any pretreatment and were exposed to the same chemotactic gradient. Random cell movement and chemokinesis (as defined in Methods) were also measured for each experiment.

As shown in Fig. 2, incubation with the anti-uPAR mAb dramatically reduces chemotaxis to $14.2 \pm 5.0 \%$ of untreated cells $(P<0.001)$. This level of cell migration was comparable with that seen in conditions of random cell movement and chemokinesis $(13.9 \pm 2.6 \%$, and $6.6 \pm 1.9 \%$ of control chemotaxis, respectively). In distinct contrast, incubation with neutralizing anti-uPA mAb had no significant effect on mononuclear cell chemotaxis (88.2 $\pm 10.3 \%$ untreated cells, $P=0.3)$. Further, saturating uPAR occupancy with exogenous HMWuPA $(4 \mu \mathrm{g} / \mathrm{ml})$ had no effect on chemotaxis $(99.9 \pm 13.4 \%$ untreated cells). Preincubating mononuclear cells with excess HMW-uPA followed by anti-uPAR mAb yielded chemotaxis that was the same as anti-uPAR treatment alone $(11.1 \pm 3.3 \%$ of untreated cells, $P<0.001$ ). Control Abs 9-49 and 99, which are isotype-type matched with $3 \mathrm{~B} 10$, but recognize unrelated monocyte antigens, had no effect on chemotaxis. From these data, we conclude that plasma membrane uPAR is required for mononuclear cell chemotaxis in response to FMLP. However, chemotaxis did not require endogenous uPA activity and was not influenced by high concentrations of exogenous catalytically active uPA.

To determine whether these effects pertained only to FMLP-induced chemotaxis, the above studies were repeated using $\mathrm{rMCP}-1(50 \mathrm{ng} / \mathrm{ml})$. As shown in Fig. 3, the results using rMCP-1 were indistinguishable from those using FMLP, demonstrating that treating cells with anti-uPAR mAb decreased 


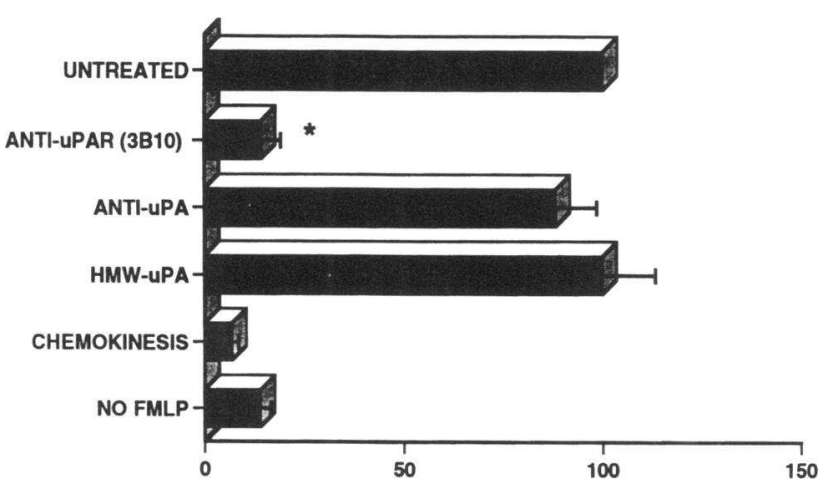

Figure 2. Effects of anti-uPAR mAb, anti-uPA mAb, and exogenous HMW-uPA on mononuclear cell chemotaxis in response to FMLP. Chemotaxis was assessed by Boyden chamber assay. Data are expressed as percentage of control (untreated cells exposed to FMLP gradient), mean \pm SEM. Controls for chemotaxis include random migration (no FMLP) and chemokinesis (equal concentrations of FMLP in upper and lower chambers). ${ }^{*} P<0.001, n=11$.

chemotaxis to $17.1 \pm 8.3 \%$ of untreated cells $(P<0.01)$. Again, preincubating cells with anti-uPA mAb had no effect on mononuclear cell chemotaxis compared with untreated cells $(97.1 \pm 24.6 \%$ of untreated cells). Similarly, the addition of HMW-uPA ( $4 \mu \mathrm{g} / \mathrm{ml})$ had no effect on mononuclear cell chemotaxis $(90.3 \pm 5.6 \%$ of untreated cells), and exogenous HMW-uPA did not affect the inhibitory action of anti-uPAR $\mathrm{mAb}(6.8 \pm 5.0 \%$ of untreated cells, $P<0.05)$. Thus, we conclude that the requirement for UPAR, but not uPA activity, is not specific to a single chemotaxin.

Despite the fact that the suppressive effects of anti-uPAR $\mathrm{mAb}$ on chemotaxis were not shared by control Abs, we wished to confirm that the effects of anti-uPAR mAb were not partly mediated by the $\mathrm{Fc}$ portion of the $\mathrm{Ab}$. We therefore examined the effects of purified anti-uPAR mAb (3B10) $F\left(a^{\prime}\right)_{2}$ fragments $\left(10 \mu \mathrm{g} / 10^{7}\right.$ cells per milliliter $)$ on chemotaxis. We found that $F\left(a b^{\prime}\right)_{2}$ fragments of the anti-uPAR mAb duplicated the effects of the intact antibody, reducing chemotaxis to $14.2 \pm 4.4 \%$ of untreated cells $(P<0.001$; Fig. 4$)$. For comparison, the $\mathrm{F}\left(\mathrm{ab}^{\prime}\right)_{2}$ fragment of anti-CD11b mAb $44\left(10 \mu \mathrm{g} / 10^{7}\right.$ cells per milliliter) did not affect chemotaxis.

We next sought to establish a dose-response relationship

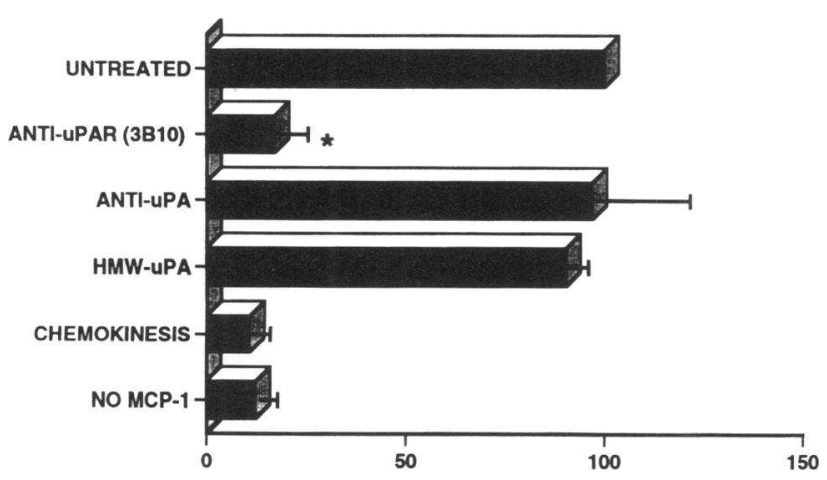

Figure 3. Effects of anti-uPAR mAb, anti-uPA mAb, and exogenous HMW-uPA on mononuclear cell chemotaxis in response to rMCP-1. Chemotaxis was assessed by Boyden chamber assay, and data are expressed as percentage of control, mean \pm SEM. ${ }^{*} P<0.01, n=3$.

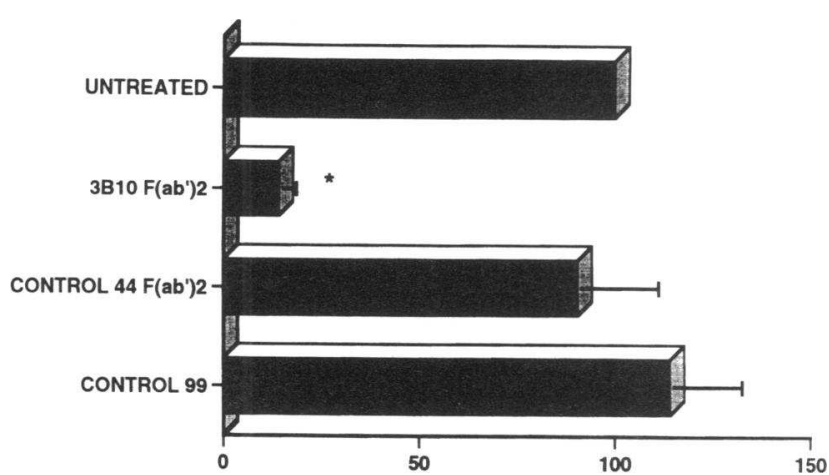

Figure 4. Effects of anti-uPAR mAb $\mathrm{F}\left(\mathrm{ab}^{\prime}\right)_{2}$ fragments on mononuclear cell chemotaxis in response to FMLP. Chemotaxis was assessed by Boyden chamber assay, and data are expressed as percentage of control, mean \pm SEM. ${ }^{*} P<0.001, n=4$.

between the concentration of anti-uPAR mAb and the suppression of chemotaxis. Dilutions of anti-uPAR ranging from 1:5 to 1:50 markedly decreased mononuclear cell chemotaxis. Chemotaxis increased to $\sim 70 \%$ of untreated cells when a 1:100 dilution was used, while lower concentrations had no significant effect. Thus, suppression of chemotaxis occurs over a broad range of $\mathrm{Ab}$ concentrations, once a threshold concentration of antibody is reached.

To determine whether other anti-uPAR Abs would duplicate the effects of 3B10 on chemotaxis, we evaluated a panel of three anti-uPAR IgM antibodies (clones 100, 109, and 68) along with an isotype-matched control mAb (anti-CD1lb clone 94). Two of the anti-uPAR antibodies appeared to suppress rMCP-1-induced chemotaxis. Chemotaxis was reduced by clone 109 to $31.4 \pm 16.1 \%$ of untreated cells $(P<0.05$; Fig. 5). Although clone 100 tended to diminish chemotaxis $(42.7 \pm 24.0 \%$ of untreated cells), the effects of this mAb were more variable and did not reach statistical significance $(P$ $<0.10)$. One anti-uPAR mAb, clone 68 , had no effect. The control antibody had no effect. These IgM mAbs recognize different epitopes and have lower binding affinity to uPAR than $3 \mathrm{~B} 10$, and this may account for the variable effect on chemotaxis.

Effects of pretreatment with $A S-u P A R$ and $A S-u P A$ on mononuclear cell chemotaxis. We next sought to block UPAR

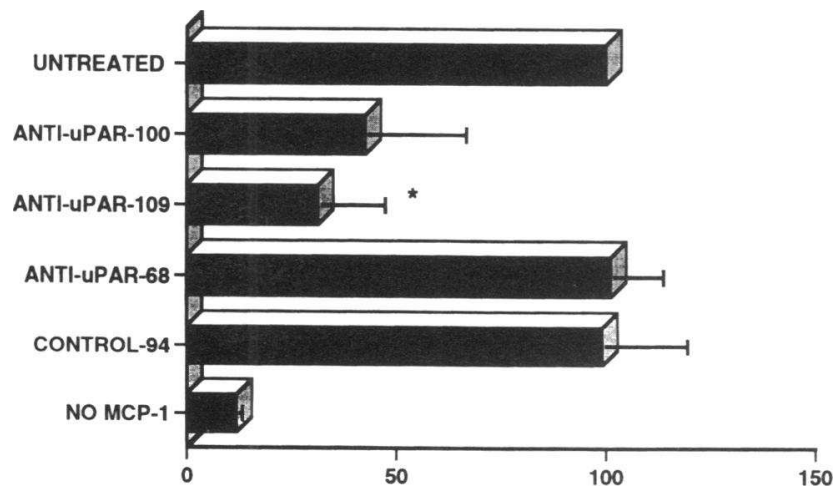

Figure 5. Effects of a panel of anti-uPAR mAb (IgM) on mononuclear cell chemotaxis in response to rMCP-1. Chemotaxis was assessed by Boyden chamber assay, and data are expressed as percentage of control, mean \pm SEM. ${ }^{*} P<0.05, n=3$. 
(-)AS-uPAR/(+)anti-uPAR Ab

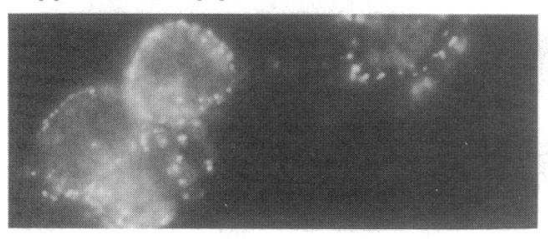

(-)AS-uPA/(+)anti-uPA Ab
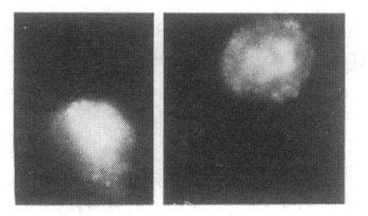

(+)AS-uPAbright fiold
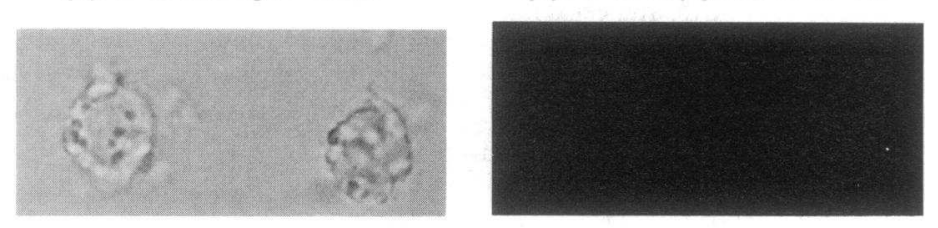

(+)AS-uPA/(+)reagent uPA/(+)anti-uPA Ab

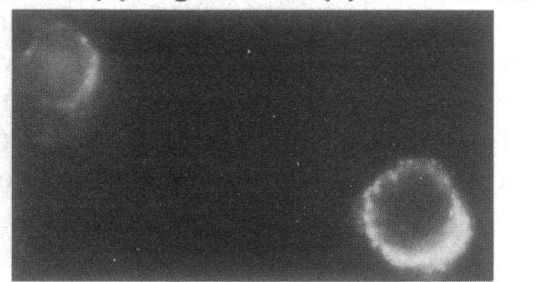

Negative Control

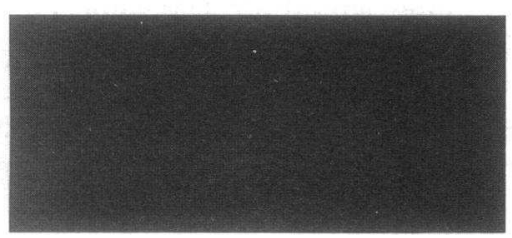

Figure 6. Fluorescent microscopy demonstrating specific suppression of surface UPA and UPAR by AS-oligos. Adherent LPS-stimulated human monocytes were incubated in media alone, with $30 \mu \mathrm{M}$ AS-uPAR, or with $30 \mu \mathrm{M}$ AS-uPA for $24 \mathrm{~h}$ at $37^{\circ}$ and indirectly immunostained with anti-uPAR or anti-uPA mAbs as indicated. ASuPAR-treated cells showed markedly diminished UPAR staining (top row). AS-uPA-treated cells showed markedly reduced staining for UPA (same field shown by light microscopy, second row). To document that ASuPA-treated cells still express uPAR capable of binding UPA, cells were immunostained for UPAR, and, in separate experiments, exogenous uPA was added, and the cells were then stained for uPA. AS-uPA treatment had no effect on cell-surface expression of UPAR (third row, right). UPAR of AS-uPA-treated cells bound exogenous uPA normally (third row, left). and UPA expression with specific antisense phosphorothioate oligonucleotides. This strategy was used to further confirm that the $3 \mathrm{~B} 10 \mathrm{mAb}$ was specifically affecting $\mathrm{UPAR}$ and to exclude the possibility that $3 \mathrm{~B} 10$ blocked chemotaxis only by steric interference with another protein closely associated with UPAR. We also reasoned that AS-oligo blockade of uPA would not only reduce uPA activity on the cell surface ( similar to anti-uPA mAb), but would also substantially reduce occupancy of uPAR by uPA. For these experiments, mononuclear cells were incubated for $24 \mathrm{~h}$ with phosphorothioate oligonucleotides in the AS orientation, encompassing highly specific sequences of UPAR and uPA mRNA, as detailed in Methods. The cells were cultured in medium alone or with AS-oligo ( 30 $\mu \mathrm{M}$ ) for $24 \mathrm{~h}$. To control for nonspecific effects of these oligonucleotides, cells were incubated in parallel with oligonucleotides of random sequence and length, S-uPAR, and NS-uPAR (as defined in Methods). Fluorescent microscopy of LPS-stimulated $(100 \mathrm{ng} / \mathrm{ml})$ monocytes was performed to confirm that we successfully blocked expression of UPAR and UPA with the relevant AS-oligo. We reasoned that if the AS-oligo was able to completely block uPAR and UPA expression despite LPS-induced upregulation of both genes, we could feel secure that the gene of interest would be blocked under basal conditions (27, 35). Monocytes were incubated with AS-uPAR or AS-uPA for
$8 \mathrm{~h}$ before LPS was added and were incubated for a total of 24 $\mathrm{h}$, followed by immunostaining and fluorescent microscopy. Negative control cells were stained with the secondary antibody alone. When cells were cultured in the absence of ASUPAR, there was bright staining for cell-surface UPAR expression (Fig. 6). However, when cells were incubated with ASuPAR for $24 \mathrm{~h}$, surface uPAR was virtually undetectable on fluorescent microscopy (Fig. 6, top row). Similarly, when cells were cultured in the absence of AS-uPA, there was bright staining for cell-surface uPA expression. However, when cells were incubated with AS-uPA for $24 \mathrm{~h}$, cell-surface uPA was markedly diminished (Fig. 6, second row). Incubating cells with AS-uPA did not block expression of uPAR (Fig. 6, third row, right), and uPAR was fully able to bind exogenous uPA (Fig. 6, third row, left).

Having confirmed that AS-oligo blockade of UPAR and uPA was effective and specific, AS-oligo-treated cells were studied in chemotaxis assays. Under all conditions, cells were $>95 \%$ viable when the chemotaxis assays were initiated. As shown in Fig. 7, cells preincubated with AS-uPAR exhibited markedly suppressed chemotaxis, to $23.2 \pm 4.0 \%$ of untreated cells $(P<0.001)$, approximating the migration seen in chemokinesis controls. Preincubating cells with AS-uPA had a minor suppressive effect on chemotaxis $(85.0 \pm 4.2 \%$ of untreated 


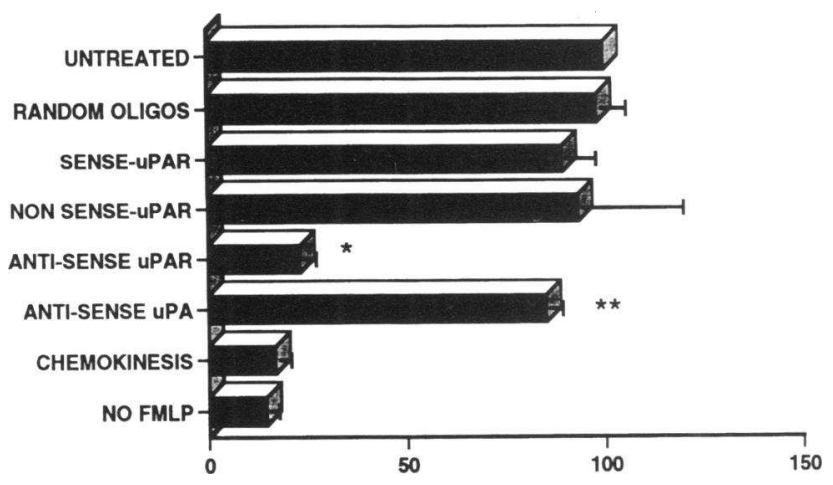

Figure 7. Effects of AS-uPAR and AS-uPA on mononuclear cell FMLP-induced chemotaxis. Mononuclear cells were pretreated with AS-uPAR, AS-uPA, or control oligos for $24 \mathrm{~h}$ before assessment of chemotaxis by Boyden chamber assay. Data are expressed as percentage of control, mean \pm SEM. ${ }^{*} P<0.001,{ }^{* *} P<0.006, n=10$.

cells, $P<0.01)$. Pretreatment with irrelevant random oligonucleotides, S-uPAR, or NS-uPAR had no effect on chemotaxis. The effect of AS-uPAR on chemotaxis was entirely reversible, as chemotaxis was fully restored to control levels 24-48 $\mathrm{h}$ after the cells were removed from AS-uPAR-containing media ( $n$ $=3$, not shown). Thus, we conclude that blocking uPAR expression on the cell surface with AS-UPAR ablates mononuclear cell chemotaxis to FMLP, while blocking uPA expression has only a relatively small effect. These observations essentially recapitulate the effects of anti-uPAR and anti-uPA mAb on chemotaxis.

\section{Discussion}

We have established that monocyte chemotaxis requires the unencumbered expression and function of plasma membrane uPAR. Binding of the anti-uPAR mAb 3B10 or ablating uPAR expression by preincubation with AS-uPAR oligo reduces migration toward a chemotactic gradient to the levels achieved by random movement or chemokinesis (Figs. 2 and 7). By contrast, blocking the catalytic site of receptor-bound uPA or suppressing uPA expression with AS-uPA oligo has little or no effect on monocyte chemotaxis (Figs. 2 and 7). Furthermore, adding exogenous HMW-uPA to increase uPAR-bound uPA has no effect on chemotaxis. These results are not specific to FMLP-induced chemotaxis, since virtually identical results were seen when rMCP-1 was used (Fig. 3). Exposure to an FMLP gradient causes UPAR and uPA to become highly concentrated at the leading edge of migration (Fig. 1). It has been shown previously that the binding sites for exogenous uPA cluster at the leading edge of chemotaxis in monocytes exposed to FMLP (10). Our observations confirm these findings by directly examining the distribution of UPAR protein itself as well as uPAR occupied with endogenously generated uPA.

It has been demonstrated conclusively that under some conditions uPA and plasmin figure importantly in the migration of mononuclear phagocytes and neoplastic cells $(3,4,36$, 37). Plasmin and uPA can degrade ECM glycoproteins, proteoglycans, and adhesion proteins, as well as facilitating destruction of collagen and elastin by activating latent matrix metalloproteinases $(6,38-41)$. Rather than examining the known ef- fects of proteolysis of stromal elements, our studies were designed to focus on the potential role of UPA and UPAR in directional movement in response to a chemotaxin. Therefore, we chose to study chemotaxis in the absence of ECM proteins and in the absence of exogenous plasminogen. Although it is likely that uPA is maintained as a single chain proenzyme in this plasminogen-free system, there is evidence that single chain UPA has enhanced catalytic activity when bound to uPAR (42). Using antibody neutralization and AS-uPA, we have shown that UPA and plasmin are not required for locomotion under matrix-free conditions. Further, we observed that high concentrations of exogenous active HMW-uPA did not affect chemotaxis or mitigate the importance of uPAR function in this process, as anti-uPAR mAb-induced blockade of chemotaxis persisted after prior treatment with HMW-uPA (Fig. 2).

Receptor-associated uPA appeared to have little role in modulating the function of UPAR in chemotaxis. Neither exogenous HMW-uPA nor neutralizing endogenously generated uPA activity with mAb had any effect on chemotaxis, but blocking uPA expression with AS-uPA did diminish chemotaxis to a small but significant degree. Therefore, it appears that uPA-mediated proteolysis does not affect chemotaxis under the conditions described, but uPA binding has at least a modest influence on uPAR function. It is likely, however, that UPA function is critical for chemotaxis under other conditions, particularly where ECM proteins are present (4).

The mechanism underlying the role of $\mathrm{UPAR}$ in cell movement remains to be elucidated. One possibility is that UPAR participates in signal transduction pathways that are required for directional locomotion. UPAR is known to transmit differentiation signals by binding UPA (23). UPAR is bound to the plasma membrane by a glycosyl phosphatidylinositol (GPI)-linkage and therefore lacks a membrane-spanning domain (26). Thus, it is likely that uPAR mediates signal transduction indirectly by associating with another surface protein that has a membrane-spanning domain. This hypothesis is particularly attractive since it has been demonstrated recently that ligand binding causes uPAR to associate with a $38-\mathrm{kD}$ partner protein that is phosphorylated on tyrosine (43). Tyrosine phosphorylation is typical of several signaling pathways associated with growth and differentiation $(43,44)$. There are other precedents for such a mechanism for signal transduction involving GPI-linked proteins. CD59, a GPI-anchored protein involved in $\mathrm{T}$ cell adhesion and activation, is tightly associated with an $80-\mathrm{kD}$ glycoprotein with protein kinase activity. Also, there are bidirectional interactions between CD16 (GPIlinked) and CD32 in neutrophils (45). Partner protein relationships were found for GPI-linked CD55 and for the ciliary neurotrophic factor receptor $(44,46-48)$. The IL-6 receptor, which contains a transmembrane segment, must combine with IL-6 and a partner protein (gp 130) for signal transduction to proceed $(49,50)$. It is possible that blocking UPAR expression or function suppresses chemotaxis by preventing a critical association between uPAR and a partner protein, thereby blocking signal transduction.

It is also possible that the obligate role of UPAR in chemotaxis is dependent upon a direct linkage between UPAR and the cytoskeleton. uPAR is known to colocalize with vinculin, $\alpha$-actinin, and actin at sites of contact between cells and ECM (14). 
This association is likely to be physiologically significant since studies using solubilized cells have shown that UPAR and vinculin reassociate even in solute phase (14). Clustering of uPAR on the plasma membrane may directly induce at least part of the cytoskeletal reorganization that is required for cell movement. Because uPAR expression is required for chemotaxis, it is certainly unlikely that cytoskeletal reorganization is causing associated uPAR to cluster merely as an epiphenomenon. Because anti-uPAR mAb-treated monocytes did not adhere or spread normally, it is also possible that UPAR is required for monocyte adhesion to surfaces as they move toward a chemotaxin. Since uPAR is GPI-linked, it is likely that UPAR and vinculin are bridged by at least one protein that contains a transmembrane segment. It is not known whether the $38-\mathrm{kD}$ partner protein of UPAR associates with the cytoskeleton (43). In neutrophils, UPAR has been shown to cocap with CR3 (CD11b/CD18). CR3 is known to enhance its proximity to actin filaments when it binds ligand (51). This observation provides a potential mechanism for an interaction of uPAR with the cytoskeleton $(52,53)$. Certainly, none of these postulated mechanisms for the role of UPAR in chemotaxis are mutually exclusive. Interactions involving cell activation, adherence, signal transduction, and motility are complex and highly interdependent. In granulocytes, exposure to chemotaxins alters plasma membrane expression of integrins, which participate in signal transduction as well as linking directly to the cytoskeleton (54-56). uPAR may likewise be multifunctional, affecting extracellular proteolysis, signal transduction, substrate attachment, and cytoskeletal reorganization in a way that culminates in directional cell movement.

The UPA-uPAR system clearly has effects on inflammatory processes that extend beyond the regulation of local plasminogen activation and matrix remodeling. uPA is able to influence directly many cell types, acting as a chemotaxin for granulocytes, a lymphocyte mitogen, and an angiogenic factor $(19,20$, 36). Plasmin can activate TGF- $\beta$ from its latent form, stimulate release of IL- 1 from $\mathrm{M} \phi$, inactivate IFN- $\gamma$, degrade $\mathrm{CD} 1 \mathrm{lb} / \mathrm{CD} 18$, and release basic fibroblast growth factor from binding sites in the $\operatorname{ECM}(22,41,57,58)$. uPA itself activates the latent form of hepatocyte growth factor-scatter factor (59). Additionally, uPA has autocrine effects, as uPA triggers differentiation of $\mathrm{M} \phi$ and promotes cellular adhesion (23). These observations, however, could not be extended to fully differentiated monocytes or alveolar macrophages. These actions were caused by uPA binding to plasma membrane uPAR by its $\mathrm{NH}_{2}$-terminal region, which contains sequences that are homologous to the receptor-binding domain of EGF. The uPAgrowth factor domain is also responsible for the growth factorlike activity seen in human osteosarcoma and squamous carcinoma cells $(60,61)$. By demonstrating that UPAR is also essential for chemotaxis, we extend the importance of UPAR beyond its established roles in anchoring UPA ectoenzyme activity and in signal transduction.

In summary, we have determined that $U P A R$ is required for monocyte chemotaxis in vitro and that this aspect of UPAR function is, in large part, independent of its ligand, uPA. By its integral role in the machinery of cellular locomotion, as well as its capacity to focus UPA- and plasmin-mediated proteolysis at the cell surface, uPAR performs two vital and distinct functions in the directional migration of mononuclear phagocytes.

\section{Acknowledgments}

The authors thank Ikuko Mizukami and Michael D'Angelo for preparation of 3B10 mAb fragments, Howard Petty for his thoughtful suggestions, and Joyce O'Brien for excellent secretarial assistance.

This work was supported by a Veterans Affairs Merit Research Grant (M. R. Gyetko), National Institutes of Health grant HL-39672 (R. G. Sitrin), and National Cancer Institute CA42246 (R. F. Todd III).

\section{References}

1. Wilkinson, P., and W. Haston. 1988. Chemotaxis: an overview. Methods Enzymol. 162:3-16.

2. Lauffenburger, D. 1991. Models for receptor-mediated cell phenomena: adhesion and migration. Annu. Rev. Biophys. Biophys. Chem. 20:387-414.

3. Danø, K., P. A. Andreasen, J. Grøndahi-Hansen, P. Kristensen, L. S. Nielsen, and L. Skriver. 1985. Plasminogen activators, tissue degradation, and cancer. Adv. Cancer Res. 44:139-266.

4. Kirchheimer, J. C., and H. G. Remold. 1989. Endogenous receptor-bound urokinase mediates tissue invasion of human monocytes. J. Immunol. 143:26342639.

5. Huber, A. R., and S. J. Weiss. 1989. Disruption of the subendothelial basement membrane during neutrophil diapedesis in an in vitro construct of a blood vessel wall. J. Clin. Invest. 83:1122-1136.

6. Liotta, L. A., R. H. Goldfarb, R. Brundange, G. P. Siegal, V. Terranova, and S. Garbisa. 1981. Effect of plasminogen activator (urokinase), plasmin, and thrombin on glycoprotein and collagenous components of basement membrane. Cancer Res. 41:4629-4636.

7. Kirchheimer, J. C., and H. G. Remold. 1989. Functional characteristics of receptor-bound urokinase on human monocytes: catalytic efficiency and susceptibility to inactivation by plasminogen activator inhibitors. Blood. 74:1396-1402.

8. Vassalli, J.-D., J.-M., Dayer, A. Wohlwend, and D. Belin. 1984. Concomitant secretion of prourokinase and of a plasminogen activator-specific inhibitor by cultured human monocytes-macrophages. J. Exp. Med. 159:1653-1668.

9. Vassalli, J.-D., A. P. Sappino, and D. Belin. 1991. The plasminogen activator/plasmin system. J. Clin. Invest. 88:1067-1072.

10. Estreicher, A., J. Mühlhauser, J.-L. Carpentier, L. Orci, and J. D. Vassalli. 1990. The receptor for urokinase type plasminogen activator polarizes expression of the protease to the leading edge of migrating monocytes and promotes degradation of enzyme inhibitor complexes. J. Cell Biol. 111:783-792.

11. Miller, M., and M. Krangel. 1992. Biology and biochemistry of the chemokines: a family of chemotactic and inflammatory cytokines. Crit. Rev. Immunol. 12:17-46.

12. Stossel, T. P. 1993. On the crawling of animal cells. Science (Wash. DC) 260:1086-1094.

13. Pöllänen, J., K. Hedman, L. S. Nielsen, K. Danø, and A. Vaheri. 1988. Ultrastructural localization of plasma membrane-associated urokinase-type plasminogen activator at focal contacts. J. Cell Biol. 106:87-95.

14. Takahashi, K., K. Ikeo, T. Gojobori, and M. Tanifuji. 1990. Local function of urokinase receptor at the adhesion contact sites of a metastatic tumor cell. Thromb. Res. (Suppl. 10):55-61.

15. Ciambrone, G. J., and P. J. McKeown-Longo. 1990. Plasminogen activator inhibitor type I stabilizes vitronectin-dependent adhesions in HT-1080 cells. J. Cell Biol. 111:2183-2195.

16. Pepper, M. S., A.-P. Sappino, R. Stöcklin, R. Montesano, L. Orci, and J.-D. Vassalli. 1993. Upregulation of urokinase receptor expression on migrating endothelial cells. J. Cell Biol. 122:673-684.

17. Liu, D., and R. Todd. 1986. A monoclonal antibody specific for a monocyte-macrophage membrane component blocks the human monocyte response to migration inhibitory factor. J. Immunol. 137:448-455.

18. Gold, L. I., A. Rostangno, B. Frangione, and T. Passalaris. 1992. Localization of the cleavage sites on fibronectin following digestion by urokinase. J. Cell. Biochem. 50:441-452.

19. Wainberg, M. A., E. Israel, and R. G. Margolese. 1982. Further studies on the mitogenic and immune-modulating effects of plasminogen activator. Immunology. 45:715-720.

20. Boyle, M. D. P., V. A. Chiodo, M. J. P. Lawman, A. P. Gee, and M. Young. 1987. Urokinase: a chemotactic factor for polymorphonuclear leukocytes in vivo. J. Immunol. 139:169-174.

21. Keski-Oja, J., J. Lohi, A. Tuuttila, K. Tryggvason, and T. Vartio. 1992. Proteolytic processing of the 72,000-Da type IV collagenase by urokinase plasminogen activator. Exp. Cell Res. 202:471-476.

22. Matsushima, K., M. Taguchi, E. J. Kovacs, H. A. Young, and J. J. Oppenheim. 1986. Intracellular localization of human monocyte associated interleukin- 
1 (IL-1) activity and release of biologically active IL-1 from monocytes by trypsin and plasmin. J. Immunol. 136:2883-2891.

23. Nusrat, A. R., and H. A. Chapman, Jr. 1991. An autocrine role for urokinase in phorbol ester-mediated differentiation of myeloid cell lines. J. Clin. Invest. 87:1091-1097.

24. Kramer, M. D., U. Vettel, M. Schmitt, J. Reinartz, G. Brunner, and A. Meissauer. 1993. Monoclonal antibodies against plasminogen activators and plasmin(ogen). Fibrinolysis. 6:103-111.

25. Min, H., R. Semnani, I. Mizukami, K. Watt, R. Todd, and D. Liu. 1992. cDNA for Mo3, a monocyte activation antigen, encodes the human receptor for urokinase plasminogen activator. J. Immunol. 148:3636-3642.

26. Mizukami, I., S. Vinjamuri, R. Trochelman, and R. Todd. 1990. A structural characterization of the Mo3 activation antigen expressed on the plasma membrane of human mononuclear phagocytes. J. Immunol. 144:1841-1848.

27. Todd, R., P. Alvarez, D. Brott, and D. Liu. 1985. Bacterial lipopolysaccharide, phorbol myristate acetate, and muramyl dipeptide stimulate the expression of a human monocyte surface antigen, Mo3e. J. Immunol. 135:3869-3877.

28. Todd, R. I., S. Meuer, P. Romain, and S. Schlossman. 1984. A monoclonal antibody that blocks class II histocompatibility related immune interactions. Hum. Immunol. 10:23-40.

29. Goldstein, S., and R. I. Todd. 1993. Mo5, a novel plasma membrane antigen synthesized by mature polymorphonuclear neutrophils: basic structural and biosynthetic features. Tissue Antigens. 41:214-218.

30. Dana, N., B. Styrt, J. Griffin, R. Todd III, M. Klempner, and M. Arnaout. 1986. Two functional domains in the phagocyte membrane glycoprotein Mol identified are monoclonal antibodies. J. Immunol. 137:3259-3263.

31. Riccio, A., G. Grimaldi, P. Verde, G. Sebastio, S. Boast, and F. Blasi. 1985. The human urokinase-plasminogen activator gene and its promoter. $\mathrm{Nu}$ cleic Acids Res. 13:2759-2771.

32. Roldan, A., M. Cubellis, M. Masucci, N. Behrendt, L. Lund, K. Dano, E. Appella, and F. Blasi. 1990. Cloning and expression of the receptor for human urokinase plasminogen activator, a central molecule in cell surface, plasmin dependent proteolysis. EMBO (Eur. Mol. Biol. Organ.). 9:467-474.

33. Stein, C. A., and J. S. Cohen. 1989. Phosphorothioate oligonucleotide analogues. In Oligodeoxynucleotides, Antisense Inhibitors of Gene Expression. J. S. Cohen, editor. CRC Press Inc., Boca Raton. 97-117.

34. Zar, J. H. 1984. Biostatistical Analysis. Prentice Hall Press, Englewood Cliffs, NJ. 121-129.

35. Chapman, H. A., Z. Vavrin, and J. B. Hibbs. 1982. Macrophage fibrinolytic activity: identification of two pathways of plasmin formation by intact cells and an inhibitor of plasminogen activator. Cell. 28:654-662.

36. Moscatelli, D., and D. B. Rifkin. 1988. Membrane and matrix localization of proteinases: a common theme in tumor cell invasion and angiogenesis. Biochim. Biophys. Acta. 948:67-85.

37. Ossowski, L., H. Russo-Payne, and E. L. Wilson. 1991. Inhibition of urokinase-type plasminogen activator by antibodies: the effect on dissemination of a human tumor in the nude mouse. Cancer Res. 51:274-281.

38. Chapman, H. A., Jr., O. L. Stone, and Z. Vavrin. 1984. Degradation of fibrin and elastin by intact human alveolar macrophages in vitro. Characterization of a plasminogen activator and its role in matrix degradation. J. Clin. Invest. 73:806-815

39. Senior, R., and S. Shapiro. 1992. Introduction: the matrix metalloproteinase family. Am. J. Respir. Cell Mol. Biol. 7:119-127.

40. Mochan, E., and T. Keler. 1984. Plasmin degradation of cartilage proteoglycan. Biochim. Biophys. Acta. 800:312-315.

41. Remold-O'Donnell, E., and B. Savage. 1988. Purification, composition, and structure of macrophage adhesion molecule. Biochemistry. 27:42-45.

42. Manchanda, N., and B. Schwartz. 1991. Single chain urokinase: augmen- tation of enzymatic activity upon binding to monocytes. J. Biol. Chem. 266:14580-14584.

43. Dumler, I., T. Petri, and W. D. Schkeuning. 1993. Interaction of urokinase-type plasminogen activator (u-PA) with its cellular receptor (uPAR) induces phosphorylation on tyrosine of a $38 \mathrm{kDa}$ protein. FEBS (Fed. Eur. Biochem. Soc.) Lett. 322:37-40.

44. Stafanova, I., V. Horejsi, I. J. Ansotegui, W. Knapp, and H. Stockinger. 1991. GPI-anchored cell-surface molecules complexed to protein tyrosine kinases. Science (Wash. DC). 254:1016-1018.

45. Hansson, G. K., L. Jonasson, P. S. Seifert, and S. Stemme. 1989. Immune mechanisms in atherosclerosis. Arteriosclerosis. 9:567-578.

46. Saksela, O., T. Hovi, and A. Vaheri. 1985. Urokinase-type plasminogen activator and its inhibitor secreted by cultured human monocyte-macrophages. J. Cell. Physiol. 122:125-132.

47. Stefanova, I., and V. Horejsi. 1991. Association of the CD59 and CD55 cell surface glycoproteins with other membrane molecules. J. Immunol. 147:1587-1592.

48. Davis, S., T. H. Aldrich, D. M. Valenzuela, V. Wong, M. E. Furth, S. P. Squinto, and G. D. Yancopoulos. 1991. The receptor for ciliary neurotrophic factor. Science (Wash. DC). 253:59-63.

49. Hibi, M., M. Murakami, M. Saito, T. Hirano, T. Taga, and T. Kishimoto. 1990. Molecular cloning and expression of an IL-6 signal transducer, gp 130. Cell. 63:1149-1157.

50. Taga, T., M. Hibi, Y. Hirata, K. Yamasaki, K. Yasukawa, T. Matsuda, T. Hirano, and T. Kishimoto. 1989. Interleukin-6 triggers the association of its receptor with a possible signal transducer, gp130. Cell. 58:573-581.

51. Davies, P. F. 1986. Vascular cell interactions with special reference to the pathogenesis of atherosclerosis. Lab. Invest. 55:5-24.

52. Petty, H. R., and T. F. Todd. 1993. Receptor-receptor interactions of complement receptor type 3 in neutrophil membranes. J. Leukocyte Biol. 54:492-494.

53. Xue, W., A. Kindzelsky, R. F. I. Todd, and H. R. Petty. 1993. Co-capping of $\mathrm{mCR} 3$ with the urokinase receptor. J. Immunol. 150:308a. (Abstr.)

54. Furfaro, S., and J. Berman. 1992. The relation between cell migration and activation in inflammation: beyond adherence. Am. J. Respir. Cell Mol. Biol. 7:248-250.

55. Zetter, B., and S. Brightman. 1990. Cell motility and the extracellular matrix. Cell Biol. 2:850-856.

56. Juliano, R. 1987. Membrane receptors for extracellular matrix macromolecules: relationship to cell adhesion and tumor metastasis. Biochim. Biophys. Acta. 907:261-278

57. Keski-Oja, J., R. M. Lyons, and H. L. Moses. 1987. Inactive secreted forms of transforming growth factor $\beta$ : activation by proteolysis. J. Cell. Biochem. Suppl. 11:60a. (Abstr.)

58. Saksela, O., and D. B. Rifkin. 1990. Release of basic fibroblast growth factor-heparin sulfate complexes from endothelial cells by plasminogen activator-mediated proteolytic activity. J. Cell Biol. 110:767-775.

59. Naldini, L., L. Tamagnone, E. Vigna, M. Sachs, G. Hartmann, W. Birchmeier, Y. Daikuhara, H. Tsubouchi, F. Blasi, and P. M. Comoglio. 1992. Extracellular proteolytic cleavage by urokinase is required for activation of hepatocyte growth factor/scatter factor. EMBO (Eur. Mol. Biol. Organ.) J. 11:4825-4833.

60. Kirchheimer, J. C., J. Wojta, G. Christ, and B. R. Binder. 1987. Proliferation of a human epidermal tumor cell line stimulated by urokinase. FASEB (Fed. Am. Soc. Exp. Biol.) J. 1:125-128.

61. Rabban, S. A., A. P. Mazar, S. M. Berneir, M. Haq, I. Bolivar, J. Henkin, and D. Goltzman. 1992. Structural requirements for the growth factor activity of the amino-terminal domain of urokinase. J. Biol. Chem. 267:14151-14156. 